\title{
Evaluación participativa del programa de educación continua en detección temprana del cáncer de mama
}

\section{Participatory evaluation of the continuing education program in early detection of breast cancer}

\author{
Devi Puerto $^{1 *}$, Luis Fontibón ${ }^{2}$, Ximena Pedraza ${ }^{3}$, Lourdes Calderón ${ }^{4}$ \\ 1 Instituto Nacional de Cancerología; dnpuerto@cancer.gov.co; https: / / orcid.org/0000-0003-0460-7769 \\ 2 Instituto Nacional de Cancerología; lffontibon@cancer.gov.co; https:/ / orcid.org/0000-0002-6635-6064 \\ 3 Hospital Universitario San Ignacio; ximenap92@hotmail.com; https: / orcid.org/0000-0002-7437-7551 \\ 4 Instituto Nacional de Cancerología; mlcalderon@cancer.gov.co; https/ / orcid.org/0000-0003-1933-3895 \\ * Correspondencia: dnpuerto@cancer.gov.co
}

Recibido: 2/8/21; Aceptado: 5/11/21; Publicado: 16/11/21

\begin{abstract}
Resumen:
Los programas de educación continua en detección temprana del cáncer son una herramienta útil y necesaria en la adquisición de conocimientos, competencias y habilidades requeridas por los profesionales de la salud. En Colombia existen falencias en la formación de profesionales en estas áreas, derivando en la falta de estandarización de técnicas y procedimientos, e inadecuada unificación de criterios de abordaje diagnóstico y terapéutico de los pacientes. El estudio buscó evaluar el programa de educación continua en detección temprana del cáncer de mama implementado por el Instituto Nacional de Cancerología entre 2016-2018. Se realizó un estudio con metodología mixta concurrente, con diseño analítico descriptivo, a partir de un análisis documental, entrevistas semiestructuradas, análisis de las encuestas de satisfacción, y de los exámenes pre y pos de las capacitaciones realizadas. Se realizó un muestreo no probabilístico y a conveniencia, la población seleccionada fueron los sujetos involucrados (profesionales capacitados, capacitadores, creadores), entre los años 2016 a 2018. Dentro de los resultados se encontró, que el nivel de satisfacción con el curso fue del $97.5 \%$; resaltando la metodología basada en el aprendizaje activo, la educación por competencias, la pertinencia de los temas abordados, la utilización de modelos anatómicos en silicona y la experticia de los capacitadores. Se encontraron diferencias estadísticamente significativas posterior a la intervención educativa $(\mathrm{V}=439 ; \mathrm{p}=0.001)$. Quedaron en evidencia barreras de tipo económico, administrativo y de divulgación; además, se sugiere implementar el curso de manera sistemática, aumentando la cobertura, divulgación y tiempo de capacitación, ofreciendo prácticas con pacientes. Concluyendo que, la implementación de programas de educación continua en profesionales de la salud son una herramienta útil y necesaria en la adquisición de competencias básicas en detección temprana del cáncer y debe contar con una estructura definida, organizada y en concordancia con los programas y políticas públicas del país.
\end{abstract}

Palabras clave: Detección Precoz del Cáncer, Neoplasias de la Mama, Educación, Evaluación de Programas y Proyectos de Salud.

\begin{abstract}
:
Continuing education programs in early detection of cancer are a useful and necessary tool for the acquisition of knowledge, competencies, and skills required by health professionals. In Colombia, there are shortcomings in the training of professionals in these areas, resulting in a lack of standardization of techniques and procedures, and inadequate unification of criteria for diagnosis and therapeutic patient management. This study sought to evaluate the continuing education program in early detection of breast cancer implemented by the National Cancer Institute of Colombia between 2016 and 2018. A concurrent mixed methodology study was conducted, with descriptive analytical design, based on a documentary analysis, semi-structured interviews, analysis of satisfaction surveys, and pretest and posttest exams. A non-probability convenience sampling was performed; the selected population consisted of subjects involved in the program (trained professionals, trainers, creators) between 2016 and 2018. Among the results, the level of satisfaction with the course was found to be $97.5 \%$, highlighting the methodology based on active
\end{abstract}


learning, competency-based education, the relevance of the topics addressed, the use of anatomical silicone models, and the expertise of trainers. Statistically significant differences were found after the educational intervention $(\mathrm{V}=439 ; \mathrm{p}=0.001)$. Economic, administrative, and dissemination barriers were evidenced. In addition, it was suggested to implement the course in a systematic way, increasing its coverage, dissemination, and training time, as well as to offer internships with patients. The study concludes that the implementation of continuing education programs among health professionals are a useful and necessary tool for the acquisition of basic skills in early cancer detection and must have a defined and organized structure in accordance with existing programs and public policies in the country.

Keywords: Early Detection of Cancer, Breast Neoplasms, Education, Program Evaluation.

\section{Introducción}

El cáncer de mama en Colombia es el primero en incidencia y mortalidad en mujeres, provocando cerca de 4411 muertes al año (1) convirtiendo a esta patología en un problema de salud pública. La aplicación de estrategias de tamizaje en algunos tipos de cáncer permite detectar la enfermedad en etapas tempranas, logrando mejores tasas de supervivencia y disminución de la mortalidad (2-3). En Colombia existen falencias en la formación de profesionales de la salud en temas de prevención y detección temprana del cáncer, adicionalmente se cuenta con escasa disponibilidad de programas organizados de educación continua que permitan fortalecer sus habilidades, competencias y destrezas en esta área, derivando en la falta de estandarización de técnicas y procedimientos, e inadecuada unificación de criterios de abordaje diagnóstico y terapéutico de los pacientes, ocasionando retrasos para el diagnóstico oportuno de esta enfermedad (4).

Uno de los objetivos de la política pública para el control del cáncer en el país, es el fortalecimiento de la gestión del talento humano (5). Debido a esto el Instituto Nacional de Cancerología ESE (INC), viene implementando un programa de educación continua dirigido a profesionales de la salud vinculados a las instituciones prestadoras de servicios de salud, para el desarrollo de sus competencias y habilidades en detección temprana del cáncer. Actualmente, el INC imparte capacitaciones presenciales y virtuales en detección temprana de diferentes tipos de cáncer, priorizados de acuerdo a su incidencia y mortalidad en el país, tales como el cáncer de mama, cuello uterino, próstata, colon y recto, piel, cáncer infantil, cáncer oral, entre otros.

La competitividad que viene alcanzando la educación y la responsabilidad que adquieren los programas de educación continua en el fortalecimiento de competencias y habilidades clínicas, hacen necesario el mejoramiento continuo de la calidad de estos. La evaluación de dichos programas puede favorecer una constante mejora de las intervenciones en este ámbito. Por lo cual, el objetivo del estudio fue evaluar el programa de educación continua en detección temprana del cáncer de mama implementado por el Instituto Nacional de Cancerología entre 2016-2018, cuyos resultados se presentan en el presente artículo.

\section{Métodos}

\section{Tipo de estudio y población}

Se llevó a cabo un estudio con metodología mixta concurrente, con diseño analíticodescriptivo; a partir de un componente cualitativo que incluyó la revisión documental del programa de educación continua en detección temprana del cáncer de mama, y la realización de entrevistas semiestructuradas; además de un componente cuantitativo enfocado en el análisis de los resultados de las encuestas de satisfacción y los resultados de 
los exámenes pre y pos, realizados a los participantes en cada una de las jornadas llevadas a cabo en la implementación del programa. Para las entrevistas se seleccionó una muestra no probabilística y a conveniencia de 17 sujetos involucrados en el programa (profesionales de la salud capacitados, capacitadores y creadores) entre los años 2016 a 2018. En relación al componente cuantitativo, se tomó como muestra la totalidad de las evaluaciones pre y post y encuestas de satisfacción diligenciadas para el periodo de estudio.

\section{Procedimiento}

Se realizó una revisión documental del programa, codificándose la información en tres grandes categorías: estructura del curso, contenidos clínicos y retroalimentación las que a su vez contenían 10 subcategorías que recogieron información relacionada con los aspectos trazadores previamente planteados (tabla 1).

Se llevaron a cabo 17 entrevistas a partir de un instrumento basado en 4 grandes temas a explorar sobre el programa: Pertinencia, barreras, estrategias evaluativas y oportunidades de mejora. La recolección se hizo a través de video llamada, solicitando el consentimiento verbal de los participantes.

Otra fuente de información fueron las encuestas de satisfacción que buscaban medir la satisfacción del usuario en términos del diseño de las capacitaciones (calidad de los expositores, aplicabilidad de los temas, pertinencia, y duración) y la logística (instalaciones, refrigerio, organización, y ayudas audiovisuales), a partir de un instrumento tipo escala Likert con 4 opciones de respuesta (entre Muy Insatisfecho a Muy Satisfecho) y un ítem para comentarios y sugerencias. Así mismo se tuvo en cuenta el resultado de las matrices de evaluación de los exámenes pre y post realizados a los participantes de las capacitaciones al inicio y finalización de la jornada.

\section{Análisis de la información}

La transcripción de las entrevistas se realizó en Word (office 2013) y el análisis a través del software NVIVO 11, la información registrada se codificó en categorías preestablecidas y emergentes, considerándose un número de referencia de al menos 2 entrevistas. Para el análisis descriptivo de las variables continuas se utilizaron medianas (con rangos como medida de variabilidad) y las variables categóricas se resumieron utilizando porcentajes. Para el análisis de los exámenes pre y pos que buscaba medir el grado de aprendizaje de los sujetos entre el periodo anterior a la intervención (pretest) y el que siguió a la misma (postest) se utilizó la prueba no paramétrica de suma de rangos de Wilconxon para muestras pareadas toda vez que la distribución de los datos no seguía una distribución normal; el programa estadístico empleado fue R-studio versión 3.6.1 y el nivel de significancia utilizado en las pruebas de hipótesis fue del 5\%. Esta investigación fue aprobada por el Comité de Ética Institucional.

\section{Resultados}

\subsection{Componente cualitativo}

A partir del análisis documental se estableció que, las estrategias de aprendizaje del programa se basaron en el enfoque de la teoría de Vygotsky, haciendo énfasis en el conocimiento como proceso social que depende de la interacción social. Este tipo de pedagogía plantea la participación guiada y escenarios de práctica, así como el plano interpersonal de los procesos de enseñanza-aprendizaje. El diseño de la metodología se 
dio bajo el dominio del aprendizaje activo, el cual incorpora principios para el aprendizaje en adultos, tales como la participación, pertinencia y practicidad.

El programa de capacitaciones tuvo sus antecedentes, a partir de un estudio piloto para la implementación de estrategias de detección temprana del cáncer de mama en Colombia, llevado a cabo por el INC, cuyos resultados mostraron una detección de hasta el doble de lesiones tempranas de mama por parte de los médicos entrenados comparados, con los no entrenados (6).

\begin{tabular}{|c|c|c|}
\hline CATEGORÍAS & SUBCATEGORÍAS & RESULTADOS \\
\hline \multirow{8}{*}{$\begin{array}{l}\text { Estructura del } \\
\text { curso }\end{array}$} & Antecedentes & $\begin{array}{l}\text { Estudio piloto para la implementación de estrategias } \\
\text { de detección temprana del cáncer de mama en } \\
\text { Colombia. }\end{array}$ \\
\hline & Diseño del programa & Enfoque de dominio del aprendizaje activo. \\
\hline & Bibliografía de soporte & $\begin{array}{l}\text { Revisión de la literatura sobre las diferentes } \\
\text { generaciones, modelos y estrategias pedagógicas. } \\
\text { Guía de práctica clínica. }\end{array}$ \\
\hline & Cursos ofrecidos & $\begin{array}{l}\text { Curso capacitación de capacitadores en detección } \\
\text { temprana en cáncer de mama. Curso en detección } \\
\text { temprana de cáncer de mama para médicos y } \\
\text { enfermeros. Curso en control de calidad de los } \\
\text { servicios de mamografía. }\end{array}$ \\
\hline & Beneficiarios & $\begin{array}{l}\text { Médicos especialistas (ginecólogos, mastólogos, } \\
\text { radiólogos), médicos generales, enfermeros/as } \\
\text { profesionales, físicos, físicos médicos e ingenieros } \\
\text { biomédicos, tecnólogos en imágenes diagnósticas. }\end{array}$ \\
\hline & Objetivos & $\begin{array}{l}\text { Desarrollo de competencias y habilidades clínicas } \\
\text { en prevención y detección temprana del cáncer, en } \\
\text { profesionales involucrados en la prevención y } \\
\text { detección temprana del cáncer de mama. }\end{array}$ \\
\hline & Metodología & Aprendizaje activo \\
\hline & Mecanismos de evaluación & $\begin{array}{l}\text { Evaluación de habilidades (mediante la aplicación } \\
\text { de guías de aprendizaje y listas de verificación) y } \\
\text { evaluación del conocimiento (exámenes pre y pos } \\
\text { curso). }\end{array}$ \\
\hline Contenidos & $\begin{array}{l}\text { Abordaje de las temáticas } \\
\text { de acuerdo al enfoque del } \\
\text { curso }\end{array}$ & $\begin{array}{l}\text { Patología mamaria benigna, Detección temprana, } \\
\text { Examen clínico de mama, Mamografía - BIRADS, } \\
\text { Diagnóstico, Tratamiento, Estrategias pedagógicas, } \\
\text { Pruebas de control de calidad en mamografía } \\
\text { Dosis Glandular media. }\end{array}$ \\
\hline $\begin{array}{c}\text { Retro- } \\
\text { alimentación }\end{array}$ & $\begin{array}{l}\text { Información suministrada } \\
\text { por los usuarios respecto a } \\
\text { aspectos relacionados con } \\
\text { las capacitaciones }\end{array}$ & $\begin{array}{l}\text { Expectativas y preocupaciones acerca del curso y la } \\
\text { detección temprana del cáncer de mama, expresadas } \\
\text { por los profesionales antes de iniciar las } \\
\text { capacitaciones. Encuestas de satisfacción. }\end{array}$ \\
\hline
\end{tabular}

Actualmente se cuenta con una oferta de cursos en modalidad virtual y presencial, dirigidos a profesionales en enfermería, medicina general, radiología, ginecología, mastología, medicina familiar entre otras especialidades, así como a técnicos y tecnólogos en radiología. Estas capacitaciones ofrecen herramientas teórico prácticas para la detección 
temprana del cáncer de mama, en temas tales como epidemiología, factores de riesgo, abordaje diagnóstico y tratamiento, además de la interpretación de los reportes de mamografía mediante la clasificación del Breast Imaging Report and Data System (BIRADS).De las 17 entrevistas realizadas, 7 fueron a enfermeras, 3 a médicos generales y 7 a médicos especialistas. La información suministrada por los participantes se codifico en 11 categorías de las cuales 5 fueron preestablecidas: Pertinencia, barreras, estrategias evaluativas y oportunidades de mejora y 6 emergentes: Antecedentes, divulgación, modalidad, práctica post curso, recursos y otros (Ver tabla 2).

\begin{tabular}{|c|c|c|}
\hline & & \\
\hline CATEGUKIA & CONTENIDO & NARRATIVA \\
\hline Antecedentes & $\begin{array}{l}\text { Hacia el año } 2011 \text { se crearon diferentes } \\
\text { cursos en respuesta a la necesidad de } \\
\text { brindar y fortalecer conocimientos en } \\
\text { detección temprana del cáncer. }\end{array}$ & $\begin{array}{l}\text { "...Ese curso nace con la necesidad de } \\
\text { brindar y fortalecer conocimientos de } \\
\text { detección temprana de cáncer de mama } \\
\text { tanto para enfermeros como para } \\
\text { médicos..." }\end{array}$ \\
\hline Divulgación & $\begin{array}{l}\text { La convocatoria de las capacitaciones } \\
\text { se realiza de manera directa con las } \\
\text { entidades de salud, universidades, } \\
\text { entes territoriales como las secretarias } \\
\text { de salud y la industria farmacéutica. }\end{array}$ & $\begin{array}{l}\text { "...la publicidad es por medio de } \\
\text { correos electrónicos, de llamadas a las } \\
\text { EPS a las IPS envíos de correos } \\
\text { electrónicos a los entes, envíos de } \\
\text { invitaciones a las asociaciones, al } \\
\text { colegio de enfermería..." }\end{array}$ \\
\hline Modalidad & $\begin{array}{l}\text { Los cursos se dictan de manera } \\
\text { presencial y a partir del } 2015 \text { se } \\
\text { incorporó la modalidad virtual, la } \\
\text { mayoría de los entrevistados tomaron } \\
\text { solamente el curso presencial y los que } \\
\text { tomaron los dos, expresan su } \\
\text { preferencia por la modalidad } \\
\text { presencial. }\end{array}$ & $\begin{array}{l}\text { "...el presencial fue muy muy bueno } \\
\text { tienes la oportunidad de preguntarle } \\
\text { al especialista ahí en ese momento tu } \\
\text { duda, que te la responda claramente, } \\
\text { en el presencial lo que te digo tienes } \\
\text { los modelos que son muy buenos y } \\
\text { pues eso no se compara a lo virtual..." }\end{array}$ \\
\hline Metodología & $\begin{array}{l}\text { Los participantes se mostraron de } \\
\text { acuerdo con la metodología empleada } \\
\text { en las capacitaciones, resaltando la } \\
\text { experticia de los capacitadores, el juego } \\
\text { de roles, los casos clínicos y la práctica } \\
\text { con modelos anatómicos }\end{array}$ & $\begin{array}{l}\text { "...me gustó mucho digamos los } \\
\text { modelos que tienen para hacer la parte } \\
\text { práctica del examen de mama, me } \\
\text { parecieron excelentes..." } \\
\text { "...me gustó, en general fue muy } \\
\text { buena y las personas que llevaron, por } \\
\text { ejemplo un doctor que era radiólogo } \\
\text { me parece que eran personas con } \\
\text { mucha competencia..." }\end{array}$ \\
\hline Contenidos & $\begin{array}{l}\text { Los contenidos de los cursos fueron } \\
\text { evaluados en términos de pertinencia, } \\
\text { aplicabilidad y actualidad, y se logró } \\
\text { evidenciar algunos elementos que } \\
\text { hicieron un aporte importante en la } \\
\text { formación del profesional capacitado, } \\
\text { tales como la sensibilización sobre la } \\
\text { situación actual del cáncer de mama en } \\
\text { Colombia y el mundo, la conducta } \\
\text { frente a la patología mamaria benigna, } \\
\text { la identificación de las pruebas de } \\
\text { tamización que existen para la } \\
\text { detección temprana del cáncer de }\end{array}$ & $\begin{array}{l}\text { "...son completos y son muy puntuales } \\
\text { y van a la realidad de las pacientes..." } \\
\text { "....yo creo que aprendí bastante, me } \\
\text { enseñaron a enfocar el paciente, a } \\
\text { distinguir patologías que a veces uno } \\
\text { queda con la duda de si es benigno o } \\
\text { es maligno..." } \\
\text { "En cuanto a si son pertinentes claro } \\
\text { que si porque cuando se habla de } \\
\text { detección temprana del cáncer de } \\
\text { mama debe hacerse énfasis primero a } \\
\text { que es el cáncer de mama y como se } \\
\text { manifiesta es lo primero que debe }\end{array}$ \\
\hline
\end{tabular}




\begin{tabular}{|c|c|c|}
\hline & $\begin{array}{l}\text { mama, y la interpretación de los } \\
\text { hallazgos mamográficos y ecográficos, } \\
\text { entre otros }\end{array}$ & $\begin{array}{l}\text { saber el profesional, se debe conocer la } \\
\text { situación de este cáncer en el país y en } \\
\text { el mundo como está la incidencia, la } \\
\text { mortalidad, la supervivencia y esas } \\
\text { cosas" }\end{array}$ \\
\hline $\begin{array}{l}\text { Estrategias } \\
\text { evaluación }\end{array}$ & $\begin{array}{l}\text { Los exámenes pretest y postest, fueron } \\
\text { la estrategia evaluativa más citada por } \\
\text { los participantes, considerada como } \\
\text { apropiada y pertinente, haciendo } \\
\text { también referencia a los simuladores, } \\
\text { casos clínicos y la utilización de listas } \\
\text { de chequeo }\end{array}$ & $\begin{array}{l}\text { "...un pretest y luego hacer un postest } \\
\text { y eso en realidad lo pone a uno en el } \\
\text { concepto de que yo no sé nada de esto, } \\
\text { pero ya después uno se da cuenta que } \\
\text { no estaba tan perdido de las cosas..." }\end{array}$ \\
\hline Recursos & $\begin{array}{l}\text { Las opiniones sobre las instalaciones y } \\
\text { recursos utilizados fueron favorables, } \\
\text { destacando las ayudas audiovisuales, } \\
\text { el uso de modelos anatómicos, la } \\
\text { experticia y dominio del tema por } \\
\text { parte del recurso humano que dirige } \\
\text { las capacitaciones. }\end{array}$ & $\begin{array}{l}\text { "...es de resaltar en el curso presencial } \\
\text { que se hace practica con modelos } \\
\text { simulando la mama..." } \\
\text { "...yo quede satisfecha, sobre todo por } \\
\text { el personal y los profesionales que } \\
\text { dieron la educación, porque se notaba } \\
\text { la preparación que tenían..." }\end{array}$ \\
\hline Práctica post curso & $\begin{array}{l}\text { La mayoría de los participantes han } \\
\text { podido poner en práctica en su } \\
\text { ejercicio profesional los conocimientos } \\
\text { adquiridos calificándolos de valiosos y } \\
\text { pertinentes, así mismo aquellos } \\
\text { profesionales que fueron capacitados } \\
\text { como capacitadores mencionaron } \\
\text { haber replicado el curso en sus lugares } \\
\text { de trabajo, aunque no de manera } \\
\text { sistemática. }\end{array}$ & $\begin{array}{l}\text { "...siempre uno lo pueda hacer en las } \\
\text { consultas, en las atenciones de los } \\
\text { pacientes..." } \\
\text { "...junto con mi hermana y otra gente } \\
\text { que fue de Risaralda nosotros } \\
\text { replicamos ese curso allá en Pereira..." }\end{array}$ \\
\hline Barreras & $\begin{array}{l}\text { Dos principales barreras fueron } \\
\text { mencionadas: } 1 \text {. La económica, al no } \\
\text { contar con un rubro fijo para realizar } \\
\text { las capacitaciones; 2. La limitada } \\
\text { difusión y publicidad }\end{array}$ & $\begin{array}{l}\text { "...el tema económico si es una barrera } \\
\text { muy importante, porque el Instituto } \\
\text { solo, no tiene cómo actuar, no tiene los } \\
\text { recursos para estar pagando al } \\
\text { personal quérita } \\
\text { exclusivamente..." } \\
\text { "...consideró que estamos en déficit de } \\
\text { difusión del curso virtual, quisiéramos } \\
\text { tener más apoyo..." }\end{array}$ \\
\hline Recomendaciones & 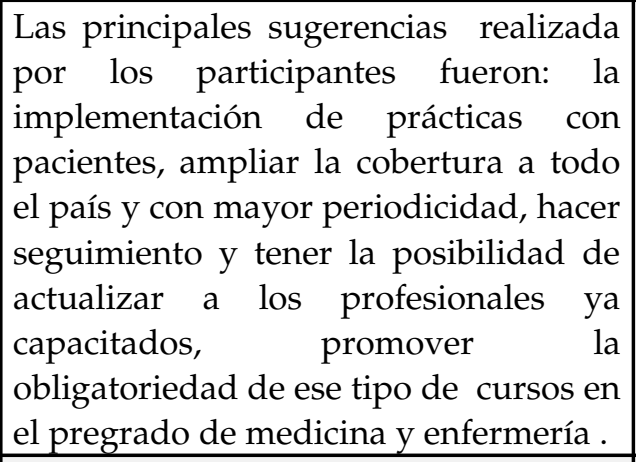 & $\begin{array}{l}\text { "...sería chévere sería si a uno le dan } \\
\text { todo el curso y hacer como una } \\
\text { práctica clínica..." } \\
\text { "...que se hicieran de pronto como más } \\
\text { seguidos ese tipo de cursos..." } \\
\text { "...hace falta que la parte práctica sea } \\
\text { un poco más larga..." }\end{array}$ \\
\hline Otros & $\begin{array}{l}\text { Un aspecto relevante que surgió, fue } \\
\text { acerca del rol de los profesionales y } \\
\text { auxiliares de enfermería en la } \\
\text { detección temprana del cáncer, } \\
\text { específicamente sobre la posibilidad y }\end{array}$ & $\begin{array}{l}\text { "...por ejemplo por norma una } \\
\text { enfermera no puede hacer digamos } \\
\text { palpación en el momento de la } \\
\text { citología... ...a mí me parece que se } \\
\text { deberían incluir los dos perfiles }\end{array}$ \\
\hline
\end{tabular}




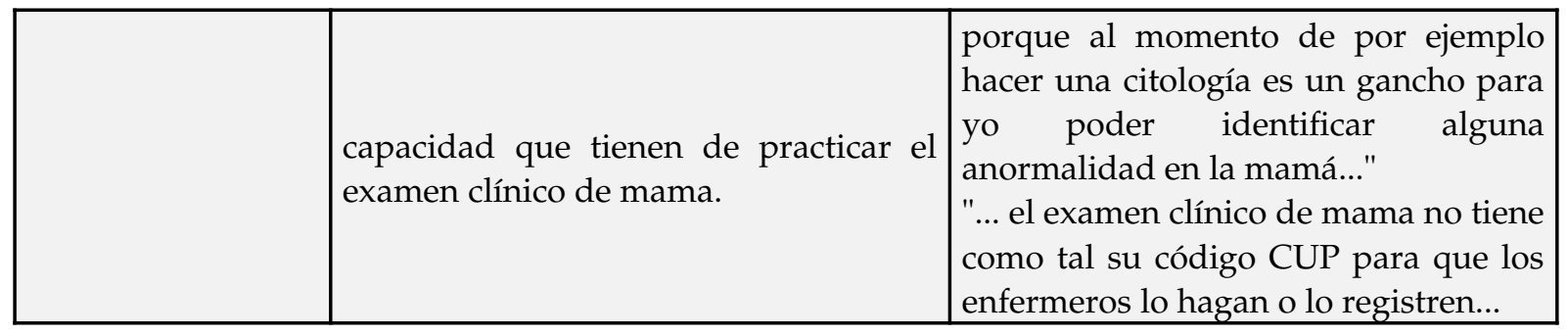

Se evidenció que los cursos presenciales cuentan con capacitadores clínicos con amplio conocimiento y experiencia en el tema, quienes realizan una evaluación y retroalimentación constante al participante sobre su progreso. Se cuenta también con expertos en pedagogía para asesorar la reproducción de la información a quienes deseen convertirse en capacitadores; además de profesionales en áreas afines a la salud pública y física médica. Fue notoria la ampliación del programa a otros beneficiarios, pues inicialmente eran solo médicos generales, y actualmente se capacitan médicos especialistas en ginecología, medicina familiar y radiología, enfermeros, físicos médicos, técnicos y tecnólogos en radiología. La gratuidad de los cursos fue un aspecto que se resaltó y que además reflejó el sentido social del INC en su misión para el control del cáncer.

La sensibilización sobre la situación epidemiológica del cáncer de mama, permitió el reconocimiento de este como un problema de salud pública. El manejo erróneo que se venía dando a algunos hallazgos en la mama, y su conducta frente a estos, además del reconocimiento de las pruebas de tamización que existen para la detección temprana, así como la interpretación de los hallazgos mamográficos y ecográficos, fueron otros contenidos que los participantes mencionaron como pertinentes y aplicables, más cuando cada día se aumenta el número de mujeres que llegan a la consulta de médica con lesiones sospechosas o exámenes radiológicos de difícil interpretación.

El material de aprendizaje de las capacitaciones se centró en manuales, modelos anatómicos de silicona, listas de chequeo, guías de aprendizaje, casos clínicos, imágenes radiológicas entre otros. Las técnicas de capacitación humanística, basadas en el uso de modelos anatómicos facilitaron el aprendizaje, reduciendo los tiempos de capacitación y el riesgo para las usuarias, permitiendo a los participantes alcanzar los niveles de desempeño en la habilidad adquirida y la pericia antes de iniciar la práctica con pacientes. La medición del progreso en el aprendizaje se efectuó a través de evaluaciones del conocimiento y habilidades.

Se identificaron barreras económicas para el desarrollo de las capacitaciones y el incremento en su cobertura. Los participantes manifestaron barreras de tipo estructural relacionadas con las restricciones que se tienen para la prescripción de imágenes diagnósticas, y la oportunidad en las citas con el especialista, que dificulta el diagnóstico oportuno. Se reconocieron oportunidades de mejora en relación a la duración de las capacitaciones, y la necesidad de contar con escenarios de práctica con pacientes, que permitan lograr una mayor apropiación de los temas por parte de los participantes. Otro aspecto a mejorar, fue la necesidad de una mayor divulgación y visibilidad del programa ante los entes gubernamentales, que reconozca la labor docente y otorgue mayor validez a esta estrategia educativa y a sus componentes.

\subsection{Componente cuantitativo}

Durante el periodo entre 2016 y 2018 un total de 418 profesionales de la salud fueron capacitados en la modalidad presencial. Se analizaron los resultados de 280 test realizados, encontrándose diferencias estadísticamente significativas entre la aplicación pre y pos test 
en los tres tipos de evaluaciones (ver tabla 3) evidenciándose puntajes más altos en los pos test reflejando el efecto del aprendizaje obtenido tanto en los conceptos básicos sobre cáncer de mama y su detección temprana, como en los conocimientos sobre las técnicas y metodologías de enseñanza. La encuesta de satisfacción fue diligenciada por 321 profesionales, mostrando un porcentaje de satisfacción del $98 \%$, con respecto al diseño y logística de las capacitaciones. Este análisis permitió identificar algunas recomendaciones a partir de la sección "Comentarios y observaciones" en relación a la duración de las capacitaciones la cual muchos consideran debería extenderse a 2 días. Finalmente, considerando la relación entre las categorías y subcategorías desde las diferentes fuentes de información, se unificaron aquellas que tenían el mismo nombre e información relacionada como: Antecedentes, metodología, estrategias de evaluación y recomendaciones; contenidos clínicos y temas abordados se unificaron bajo el nombre de temáticas, y se incluyeron los análisis cuantitativos dentro del análisis global (figura 1).

Tabla 3. Resultados del análisis cuantitativo. Comparación de los pre y post test.

\begin{tabular}{|c|c|c|c|c|}
\hline \multirow[t]{2}{*}{ Evaluación } & $N(\%)$ & \multicolumn{2}{|c|}{ Mediana(RI) } & Suma de rangos de Wilconxon \\
\hline & Total $=280$ & Pre & Post & \\
\hline Médicos generales y enfermeras ${ }^{a}$ & $175(62.5)$ & $8(3)$ & $12(3)$ & $\mathrm{V}=439 ; \mathrm{p}$-value $<0.001^{*}$ \\
\hline Especialistas $^{b}$ & $70(25)$ & $12.50(4)$ & $16(2)$ & $\mathrm{V}=73 ; \mathrm{p}$-value $<0.001^{*}$ \\
\hline Capacitación a capacitadores ${ }^{c}$ & $35(12.5)$ & $11(4)$ & $14(2)$ & $\mathrm{V}=73 ; \mathrm{p}$-value $<0.001^{*}$ \\
\hline
\end{tabular}

${ }^{a}$ Test cuyo puntaje máximo es $15 ;{ }^{\mathrm{b}}$ Test cuyo puntaje máximo es 18 ; $^{\mathrm{c}}$ Test cuyo puntaje máximo es 17 ; *Diferencias estadísticamente significativas.

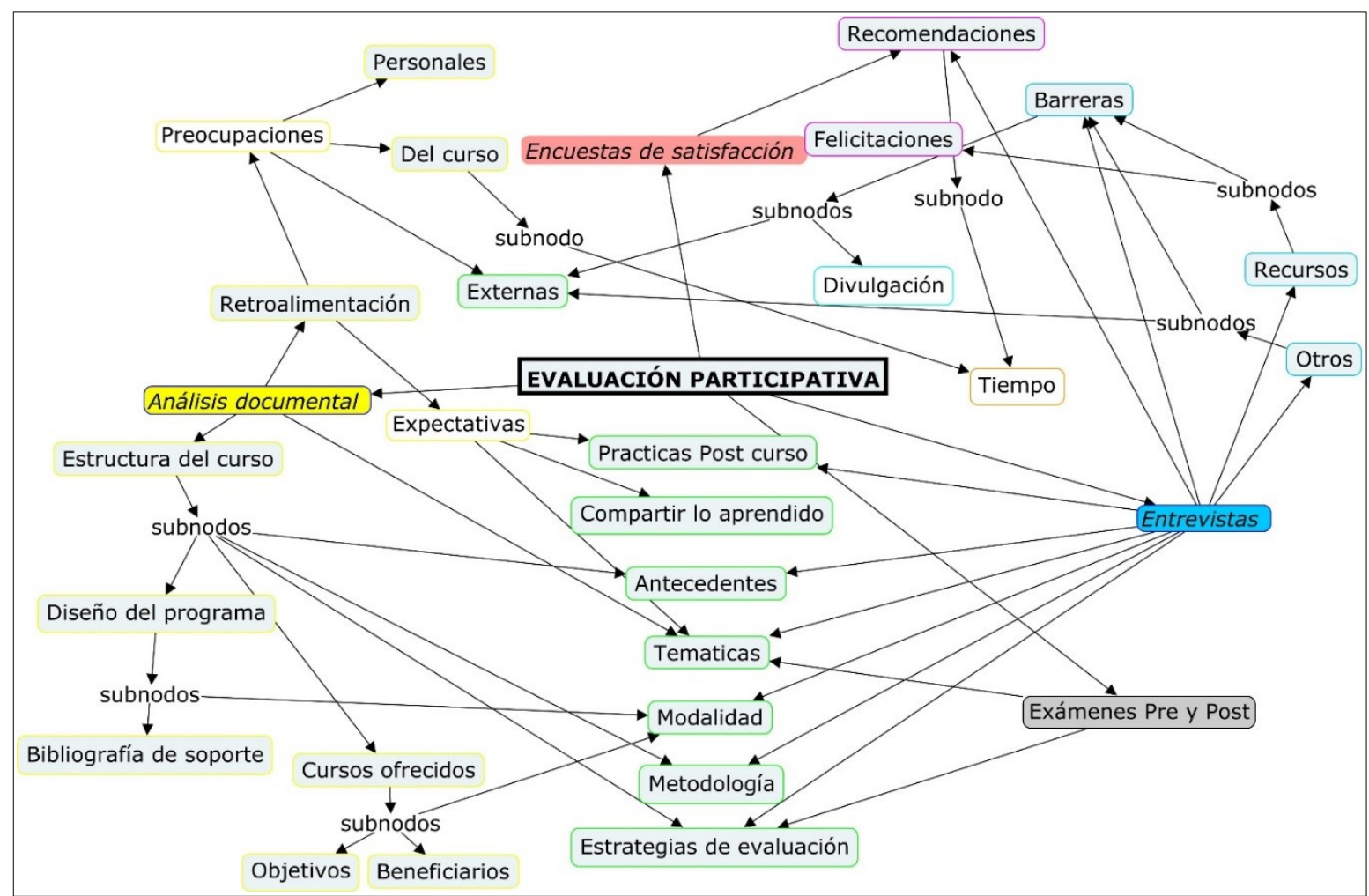

Figura 1. Mapa categorial: Sombreadas en colores, las fuentes primarias de información para el análisis (Análisis documental, encuestas de satisfacción, exámenes pre y post y entrevistas) con sus respectivas categorías y subcategorías (indicadas por las flechas). 


\section{Discusión}

El programa de educación continua ha sido una estrategia bien recibida por parte de los profesionales de la salud como herramienta para el manejo del cáncer de mama. Se evidenció un incremento estadísticamente significativo en los conocimientos de los participantes, resultados similares a los encontrados por otros autores (7-11), quienes emplearon instrumentos de evaluación semejantes a los utilizados en las capacitaciones (exámenes pre y post), quienes resaltaron la importancia de este tipo de actividades para la adquisición y fortalecimiento de competencias en detección temprana del cáncer de mama, puesto que se ha observado un escaso conocimiento en cuanto a factores de riesgo, signos y síntomas, y métodos de diagnóstico en profesionales de la salud (12), así como falencias en la enseñanza de técnicas y procedimientos en los programas curriculares de pregrado de medicina y enfermería (13-14). No obstante, Toftegaard et al, encontraron que la educación médica continua tiene un efecto limitado en el conocimiento sobre el diagnóstico del cáncer, pues al utilizar un grupo control, observaron leves diferencias entre los test pre y pos dentro de cada grupo (experimental y control) pero no encontraron diferencias al compararlos entre ellos (15); a diferencia del estudio llevado a cabo por Ceber et al, en el que si se encontraron diferencias dentro y entre los grupos al utilizar un grupo que no recibió educación (16); por otro lado, varios autores han encontrado diferencias intra grupo pero no entre grupos al comparar diferentes modalidades de enseñanza, como virtual, presencial, por mensaje de texto, entre otras (17-19); lo cual apunta a que los programas de educación continua sin importar la modalidad son útiles y tienen un efecto positivo en el aprendizaje de las competencias requeridas en la detección temprana del cáncer de mama.

En relación a los temas abordados en las capacitaciones, se observó aprobación y satisfacción por parte de los participantes considerándolos útiles y oportunos; encontrando estos resultados coherentes con aquellos mencionados en la literatura sobre programas de educación continua en detección temprana (20-21), dentro de los cuales predominan, contenidos tales como la epidemiología, patología mamaría benigna, diagnóstico y tratamiento del cáncer, signos y síntomas, así como la implementación de estrategias de tamización mediante el examen clínico de mama y la mamografía (22) en los cuales se basan las capacitaciones del programa. Así mismo, la utilización de modelos anatómicos en silicona, que han demostrado su utilidad en la enseñanza del examen clínico de mama(23-26), fue una de las estrategias más destacadas por los participantes junto con la experticia y disposición de los capacitadores. Hallazgos encontrados por Karayurt et al, van en la misma dirección de este estudio, señalando la necesidad de aumentar el tiempo de duración de los entrenamientos (10). Por otro lado, se destaca el papel del profesional de enfermería en la detección temprana del cáncer de mama, puntualmente en la necesidad y oportunidad de dichos profesionales en la realización del examen clínico a las usuarias que entren en contacto con los servicios de salud. La falta de recursos financieros, así como falencias en la promoción de los cursos fueron algunas de las barreras mencionadas y que no difieren de las ya expuestas con anterioridad en la literatura (27), siendo necesario crear para cada una de ellas acciones de mejora dirigidas a superar tales dificultades. En su mayoría la evaluación a programas de educación continua se centran en el análisis comparativo de los exámenes escritos pre y post, otros incorporan la percepción y evaluación que hacen los participantes del curso (28) y algunos van más allá involucrando a otros actores implicados como administradores, proveedores de servicio y pacientes (29-30) y quizá lo que representa un mayor reto, es la evaluación de la práctica de los profesionales capacitados, tales como la cantidad de mamografías solicitadas, exámenes clínicos realizados y hasta la proporción de diagnósticos en estadios 
tempranos (29-31). Cada uno de estos aspectos es primordial para lograr un análisis más completo y global de este tipo de programas educativos.

Esta investigación presentó algunas limitaciones, como no contar con la totalidad de registros de los test para la medición del efecto de las capacitaciones. Además, la fragmentación de la información requirió un mayor tiempo de recolección de la misma. Sin embargo, esta evaluación evidenció elementos que permitirán fortalecer la implementación del programa, y servirá como base para futuras investigaciones en el ámbito de la educación dirigida a los proveedores de salud.

\section{Conclusiones}

- La implementación de programas de educación continua en profesionales de la salud son una herramienta útil y necesaria en la adquisición de competencias básicas en detección temprana del cáncer y debe contar con una estructura definida, organizada y en concordancia con los programas y políticas públicas del país.

- El diseño y estructura del programa son acordes a las necesidades de atención de cáncer en el país y a las rutas de atención desde la prevención, detección temprana, diagnóstico y tratamiento, enfocado en contenidos, materiales y estrategias evaluativas actuales, aplicables y pertinentes.

- Las barreras identificadas son primordialmente de índole económico, ya que la ejecución de cada curso conlleva gastos importantes.

- Los efectos de la intervención educativa en el conocimiento fueron relevantes, observándose aumento significativo en los puntajes postest; no obstante, es necesario realizar evaluaciones a largo plazo en relación a la adherencia de las recomendaciones entregadas.

- Se identificaron oportunidades de mejora, relacionadas principalmente con el tiempo de duración del curso y la divulgación del mismo.

Contribuciones de los autores: Todos los autores contribuyeron en el diseño del estudio, recolección de información y posterior planteamiento del manuscrito.

Financiación: No ha habido financiación.

Agradecimientos: A docentes y usuarios del programa de educación.

Declaración de conflicto of interés: Los autores declaran no tener ningún conflicto de intereses.

\section{Referencias}

1. Global Cancer Observatory [Sitio de internet]. 2020. [Consultado 2021 may 2] Disponible en: https://gco.iarc.fr/today/data/factsheets/populations/170-colombia-fact-sheets.pdf

2. Villarreal-Garza C, García-Aceituno L, Villa AR, Perfecto-Arroyo M, Rojas-Flores M, LeónRodríguez E. Knowledge About Cancer Screening Among Medical Students and Internal Medicine Residents in Mexico City. J Cancer Educ 2010;25(4):624-631. https://doi.org/10.1007/s13187-010-0098-6

3. Sundmacher L, Busse R. The impact of physician supply on avoidable cancer deaths in Germany. A spatial analysis. Health Policy Educ 2011;103(1):53-62. https://www.sciencedirect.com/science/article/pii/S0168851011001618

4. Wisner C, Salazar L, Puerto D, Cotes J. Programa de educación continua para profesionales en salud para la prevención y detección temprana del cáncer en Colombia. Experiencia del 
Instituto Nacional de Cancerología. Rev Monit Estrateg Supt Nac Salud 2013;4:78-83. https://docs.supersalud.gov.co/PortalWeb/Comunicaciones/MonitorEstrategico/MonitorEstrategico004-2013.pdf\#page $=79$

5. Ministerio de Salud y Protección Social, Instituto Nacional de Cancerología. Plan decenal para el control del cáncer en Colombia, 2012-2021. Bogotá, Colombia; 2012. 1-124 p.

6. Murillo R, Díaz S, Perry F, Poveda C, Piñeros M, Sánchez O, et al. Increased breast cancer screening and downstaging in Colombian women: A randomized trial of opportunistic breastscreening. Int J Cancer 2016;138(3):705-713.

7. Pace LE, Dusengimana J-MV, Keating NL, Hategekimana V, Rugema V, Bigirimana JB, et al. Impact of Breast Cancer Early Detection Training on Rwandan Health Workers' Knowledge and Skills. J Glob Oncol 2018;(4):1-10.

8. Bittencourt L, Scarinci IC. Training community health workers to promote breast cancer screening in Brazil. Health Promot Int 2019;34(1):95-101.

9. Edwards QT, Seibert D. Pre- and posttest evaluation of a breast cancer risk assessment program for nurse practitioners. J Am Acad Nurse Pract 2010;22(7):376-381.

10. Karayurt Ö, Gürsoy AA, Taşçı S, Gündoğdu F. Evaluation of the breast cancer train the trainer program for nurses in Turkey. In: Journal of Cancer Education 2010. p. 324-328.

11. Rick TJ, Deming CM, Helland JR, Hartwig KA. Cancer Training for Frontline Healthcare Providers in Tanzania. J Cancer Educ 2017;34(1):111-115.

12. Heena H, Durrani S, Riaz M, Alfayyad I, Tabasim R, Parvez G, et al. Knowledge, attitudes, and practices related to breast cancer screening among female health care professionals: A cross sectional study. BMC Womens Health 2019;19(1):1-11.

13. Fernández-Deaza GP, Villate-Soto SL, Puerto-Jiménez DN. Educación basada en competencias para estudiantes de medicina sobre la prevención y detección temprana del cáncer. Educ Medica 2017;18(4):270-275. http:/ /dx.doi.org/10.1016/j.edumed.2016.11.011

14. González-Robledo MC, González-Robledo LM, Caballero M, Aguilar-Martínez ME. Formación de médicos y enfermeras para la detección temprana del cáncer de mama en México. Rev Salud Publica 2011;13(6):966-979.

15. Toftegaard BS, Bro F, Falborg AZ, Vedsted P. Impact of continuing medical education in cancer diagnosis on GP knowledge, attitude and readiness to investigate - A before-after study. BMC Fam Pract 2016;17(1):1-10. http://dx.doi.org/10.1186/s12875-016-0496-x

16. Ceber E, Turk M, Ciceklioglu M. The effects of an educational program on knowledge of breast cancer, early detection practices and health beliefs of nurses and midwives. J Clin Nurs 2010;19(15-16):2363-2371.

17. Bell RA, McDermott H, Fancher TL, Green MJ, Day FC, Wilkes MS. Impact of a randomized controlled educational trial to improve physician practice behaviors around screening for inherited breast cancer. J Gen Intern Med 2015;30(3):334-341.

18. Alipour S, Jannat F, Hosseini L. Teaching breast cancer screening via text messages as part of continuing education for working nurses: A case-control study. Asian Pacific J Cancer Prev 2014;15(14):5607-5609.

19. Heydari E, Noroozi A. Comparison of two different educational methods for teachers' mammography based on the health belief model. Asian Pacific J Cancer Prev 2015;16(16):69816986.

20. Brownson RC, Ballew P, Kittur ND, Elliott MB, Haire-Joshu D, Krebill H, et al. Developing competencies for training practitioners in evidence-based cancer control. J Cancer Educ 2009;24(3):186-193.

21. Magaña-Valladares L, González-Robledo MC, Rosas-Magallanes C, Mejía-Arias MÁ, ArreolaOrnelas H, Knaul FM. Training primary health professionals in breast cancer prevention: Evidence and experience from Mexico. J Cancer Educ 2018;33(1):160-166.

22. Albeshan SM, Hossain SZ, Mackey MG, Brennan PC. Can Breast Self-examination and Clinical Breast Examination Along With Increasing Breast Awareness Facilitate Earlier Detection of Breast Cancer in Populations With Advanced Stages at Diagnosis? Clin Breast Cancer 2020;In press:1-7. https://doi.org/10.1016/j.clbc.2020.02.001 
23. Madan AK, Aliabadi-Wahle S, Babbo AM, Posner M, Beech DJ. Education of medical students in clinical breast examination during surgical clerkship. Am J Surg 2002;184(6):637-641.

24. Iannotti RJ, Finney LJ, Sander AA, De Leon JM. Effect of clinical breast examination training on practitioner's perceived competence. Cancer Detect Prev 2002;26(2):146-148.

25. Angarita FA, Price B, Castelo M, Tawil M, Ayala JC, Torregrossa L. Improving the competency of medical students in clinical breast examination through a standardized simulation and multimedia-based curriculum. Breast Cancer Res Treat 2018;173(2):439-445. http://dx.doi.org/10.1007/s10549-018-4993-6

26. Steiner E, Austin DF, Prouser NC. Detection and description of small breast masses by residents trained using a standardized clinical breast exam curriculum. J Gen Intern Med 2007;23(2):129134.

27. Aribal E, Mora P, Chaturvedi AK, Hertl K, Davidović J, Salama DH, et al. Improvement of early detection of breast cancer through collaborative multi-country efforts: Observational clinical study. Eur J Radiol 2019;115(January):31-38. https://doi.org/10.1016/j.ejrad.2019.03.020

28. Dixon H, Hordern A, Borland R. The breast cancer distance education program. Cancer Nurs 2001;24(1):44-52.

29. Basu P, Selmouni F, Belakhel L, Sauvaget C, Abousselham L, Lucas E, et al. Breast Cancer Screening Program in Morocco: Status of implementation, organization and performance. Int J Cancer 2018;143(12):3273-3280.

30. Torres E, Richman AR, Schreier AM, Vohra N, Verbanac K. An Evaluation of a Rural Community-Based Breast Education and Navigation Program: Highlights and Lessons Learned. J Cancer Educ 2017;34(2):277-284.

31. Dietrich AJ, O'Connor GT, Keller A, Carney PA, Levy D, Whaley FS. Cancer: Improving early detection and prevention. A community practice randomised trial. Br Med J 1992;304(6828):687691.

(C) 2021 Universidad de Murcia. Enviado para su publicación en acceso abierto bajo los términos y condiciones de la licencia Creative Commons Reconocimiento-NoComercial$\begin{array}{lllll}\text { Sin } & \text { Obra } & \text { Derivada } & 4.0 & \text { España }\end{array}$ (http:/ / creativecommons.org/licenses/by/4.0/). 